\title{
Influence of Nanoadditives on Rheological Properties of Fuel Oil
}

\author{
Elvira R. Zvereva \\ Kazan State Power Engineering University \\ Kazan, Russia \\ 6elvira6@list.ru \\ Railya V. Khabibullina \\ Kazan State Power Engineering University \\ Kazan, Russia \\ zinnatullina-rai@mail.ru
}

\author{
Gulnara R. Akhmetvalieva \\ Kazan State Power Engineering University \\ Kazan, Russia \\ gu_94@mail.ru
}

\author{
Anastasiya O. Makarova \\ Kazan State Power Engineering University, Kazan Institute of \\ Biochemistry and Biophysics, KazSC RAS, Kazan, Russia \\ tat355@mail.ru
}

\author{
Olga S. Zueva \\ Kazan State Power Engineering University \\ Kazan, Russia \\ ostefzueva@mail.ru
}

\begin{abstract}
The influence of nanoadditives of organic and inorganic origin on the rheological properties of heavy fuel oil was considered. Diproxamine, an oil soluble nonionic surfactant, multiwalled carbon nanotubes of the carbon nanomaterial Taunit and dehydrated carbonate sludge were used as additives. The search for optimal concentrations of the applied nanoadditives and the values of the change in the viscosity of the resulting composite fuel was carried out. The authors' studies of the fuel oil samples viscosity have shown the possibility of improving its rheological properties by introducing nanostructured additives diproxamine $(0.5 \mathrm{wt}$. \%), carbon nanotubes of the carbon nanomaterial Taunit $(0.0125$ wt. \%) or dehydrated carbonate sludge (0.1 wt. \%). The observed change in viscosity was $10-20 \%$. It has been established that diproxamine improves viscosity properties of fuel at sufficiently high temperatures $\left(75^{\circ} \mathrm{C}\right)$, and its joint application with nanotubes is effective even at lower temperatures $\left(65^{\circ} \mathrm{C}\right)$. It was shown that the use of dehydrated carbonate sludge also leads to a decrease in the boiler fuel viscosity. The existence of a synergistic effect in the joint application of CNTs with carbonate sludge has been established. Possible mechanisms for changing the viscosity properties of fuel were considered.
\end{abstract}

Keywords-fuel oil, carbon nanotubes, viscosity, surfactant

\section{INTRODUCTION}

In connection with the deepening of oil refining in recent years, there has been a tendency of deterioration of the properties of boiler fuel due to an increase in the proportion of tar and heavy petroleum products in fuel oil. Reducing the quality of fuel oil for the consumer means a deterioration in its physical, chemical and operational characteristics, which leads to increased fuel transportation and storage costs and has a significant impact on the operation of the main and auxiliary equipment of thermal power plants and boiler houses, as well as the volume of harmful substances, emitted to the atmosphere [1].

The need to solve interrelated problems of fuel economy, to improve technical and economic indicators of boiler units, to reduce emissions of harmful substances into the atmosphere requires the search for efficient and inexpensive methods, one of which is the addition of special additives to the fuel $[2,3]$. Recently, when creating unconventional additives to oils and fuels, methods and materials of nanotechnology are used, including functional nanoscale structures of organic and inorganic origin [4-10]. The use of additives can lead to a decrease in the viscosity of the fuel, contributing to a reduction in energy costs, when it is pumped through pipelines, unloading from cisterns and supply to the boiler, and therefore is of fundamental importance for energy conservation purposes.

In this paper [11], it was shown that a nonionic surfactant, diproxamine, can be used as an additive to a heavy fuel oil and for dispersing nanoparticles. It is generally used in the petroleum and petroleum refining industry as a non-ionic demulsifier and a paraffin deposition inhibitor or as a demulsifying additive for lubricants, particularly turbine oils [12].

In addition, it has been found that small additions of nanoparticles of any nature, including carbon nanotubes (CNTs), can be used to improve the rheological properties of liquid heterogeneous systems, which use fuel oil [13-16]. To explain the effects observed in heterogeneous systems, various mechanisms of viscosity decrease were proposed, which explained the change in the morphology of the composite and the transition to stratified flow $[17,18]$. It was noted that the greatest effect of viscosity reduction is observed at a certain concentration of nanoparticles, which can be found by 
studying the concentration curves of the viscosity dependences of the liquid systems under consideration $[16,19]$.

All of the above-mentioned facts give grounds for studying the possibilities of using carbon nanomaterials in order to improve the operational properties of organic boiler fuel, including other nanostructured additives that have already proved their effectiveness. It should be noted that carbon nanotubes, despite being non-biodegradable nanoparticles, are completely burned together with fuel, since they consist of pure carbon, and their catalytic properties lead to more complete combustion of other components of the fuel, which may be important for eliminating negative consequences of heavy fuel use. In the world literature there are results on the use of carbon nanotubes as nanoadditives to motor fuel [2024]. As an additive to heavy fuel oil, we used carbon nanotubes for the first time.

To improve the physicochemical properties of organic fuel, to increase the efficiency and environmental friendliness of combustion and in order to save it, in addition to the use of carbon nanotubes, there has been a tendency to add to the fuel the functional nanoscale structures - metal nanoparticles, oxides, carbides, nitrides. Nanoparticles of aluminum, iron, boron, manganese, magnesium, copper, calcium and cerium, as well as oxides of aluminum, zinc, manganese, copper and all lanthanides are effective for diesel fuel [4-10]. The authors' studies have shown that the use of expensive nanoparticles as additives to heavy hydrocarbon fuels can to some extent be replaced with additives of dehydrated carbonate sludge, which is a waste of the process of chemical water purification of thermal power plants. The purpose of this work was to investigate the possibilities of improving the rheological properties of fuel oil by adding carbon nanotubes and other nanostructured formations, and to determine the optimum concentrations of the nanoadditives used and the magnitude of the change in the viscosity of the resulting composite fuel.

\section{MATERIALS}

Samples of high-sulfur M100 brand fuel oil, produced by the Nizhnekamsk oil refinery, used at the Kazan thermal power plant as an emergency and backup fuel, were taken as boiler fuel for the studies. According to the structural composition, the M100 brand fuel oil mainly consists of paraffin (49.2\%) and aromatic hydrocarbons (42.6\%). It should be noted that when studying the viscosity of fuel oil samples and water-fuel emulsions with additives prepared based on M100 brand boiler oil, the authors encountered fundamentally different types of viscosity dependence for specific fuel oil samples, taken from different sources at different times. Even within the framework of a single brand, the samples can demonstrate both the viscosity values that are almost 2 times different, and the different rheological behavior depending on the gradient of the rate of layer migration. This fact undoubtedly complicates the description of the processes, occurring when nanostructured formations are added to the fuel, introduces its own corrections to the results reproducibility and prevents the selection of optimal compositions of various additives.
Diproxamine (the production of KAZANORGSINTEZ, http://www.kazanorgsintez.ru) was used as an additive to heavy fuel oil and for dispersing carbon nanotubes. Diproxamine is a nitrogen-containing block copolymer. The production method is based on the interaction of ethylenediamine with propylene oxide, and further on with ethylene oxide and subsequent propylene oxidation of obtained polymer. It is an oil-soluble non-ionic surfactant and one of the universal domestic demulsifiers. The average molecular weight of polymer molecules of diproxamine is 4500-5000. By their size and structure, diproxamine molecules are somewhat similar to nanoparticles. The structural formula of diproxamine is presented in figure 1.

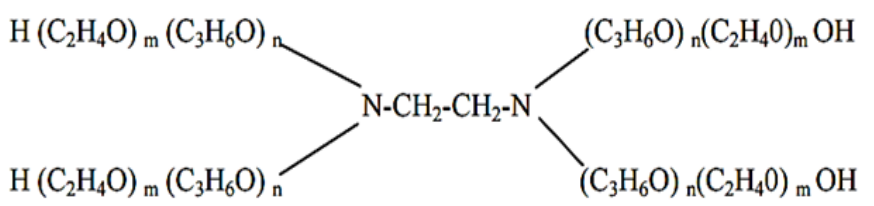

$\mathrm{n}: \mathrm{m}=70: 30$

Fig. 1. Structural formula of diproxamine - a nitrogen-containing block copolymer on the base of ethylenediamine

Multi-walled carbon nanotubes (CNTs) of the carbonaceous nanomaterial "Taunit" (http://www.nanotc.ru), dispersed in diproxamine, were used as nanosize additives. A carbonaceous nanomaterial "Taunit" is a set of nanofibers (the multi-walled nanotubes are mainly formed with the conic shape graphene layers) with a length of up to 2 and more $\mu \mathrm{m}$ and an external diameter, usually varying from 15 to $40 \mathrm{~nm}$. Basic physicochemical indicators of the carbonaceous nanomaterial "Taunit" are presented in Table I.

TABLE I. PHYSICOCHEMICAL INDICATORS OF THE CARBONACEOUS NANOMATERIAL "TAUNIT"

\begin{tabular}{|c|c|}
\hline $\begin{array}{c}\text { Physicochemical indicators of } \\
\text { the carbonaceous nanomaterial }\end{array}$ & Nanomaterial \\
\cline { 2 - 2 } & "Taunit" \\
\hline External diameter, $\mathrm{nm}$ & $20-70$ \\
\hline Internal diameter, $\mathrm{nm}$ & $5-10$ \\
\hline Length, $\mu \mathrm{m}$ & $\geq 2$ \\
\hline Total impurities (primary), & $\leq 5$ \\
(after cleaning) & $\leq 1$ \\
\hline Bulk density g $\mathrm{cm}^{-3}$ & $0.4-0.6$ \\
\hline Specific surface area, $\mathrm{m}^{2} \mathrm{~g}^{-1}$ & $120-130$ \\
\hline Thermal stability in air, ${ }^{\circ} \mathrm{C}$ & $\leq 600$ \\
\hline
\end{tabular}

The initial carbon nanomaterial consists of agglomerates of multi-walled nanotubes - tangles of filamentous formations, in which carbon nanotubes are interconnected by the physical forces of Van der Waals. The SEM image of this probe of carbonaceous nanomaterial "Taunit", dispersed in pure water, is shown in figure 2.

To uniformly distribute nanotubes throughout the sample volume, they were dispersed using various surfactants. The authors' earlier experiments on the study of the rheological properties of fuel oil with the addition of carbon nanotubes, dispersed in aqueous solutions of anionic surfactant - sodium 
dodecyl sulfate, proved the existence of a decrease in viscosity when a sufficiently large amount of CNTs (0.82 wt. \%) was introduced to fuel [25]. Use of carbon nanotubes at such concentrations is unprofitable.

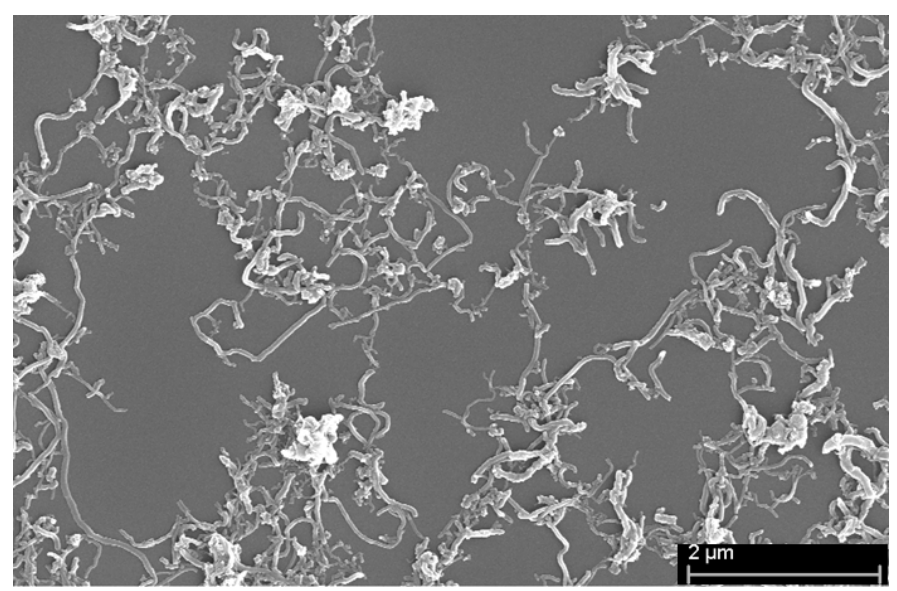

Fig. 2. SEM image of the suspension of carbonaceous nanomaterial "Taunit" dispersed in pure water

Therefore, the oil-soluble nonionic surfactant diproxamine was chosen as a more suitable dispersion medium for CNTs. The choice of this surfactant for the dispersing of CNTs is also due to the fact that the use of diproxamine as an additive to heavy fuel oil can in some cases lead to an improvement in its rheological characteristics. When dispersing carbon nanotubes in diproxamine, $1 \mathrm{~g}$ of CNTs was mixed with $40 \mathrm{ml}$ of diproxamine, then the sample was subjected to a $15-\mathrm{kHz}$ ultrasonic treatment in a water bath of ultrasonic disperser Bandelin SONOREX TK52 (Germany) for 15 minutes at $40{ }^{\circ} \mathrm{C}$. Further, the prepared suspension was dissolved in fuel samples at a given concentration.

The choice of carbonate sludge as another additive, which the authors investigated, was dictated by their earlier good results on improving the rheological properties of fuel oil in its use [11]. Carbonate sludge is formed in the process of coagulation and liming of natural waters at thermal power plants and, from the production point of view, it is a waste. Carbonate sludge as a product, obtained by chemical precipitation, has a complex of specific physical and chemical properties, among which a diverse chemical composition (calcium carbonates, magnesium hydroxides and iron, aluminum compounds, etc.), as well as its high dispersity and surface activity. The compounds, entering into its composition in the process of sludge formation, create a nano- and microdimensional structure originally separated by water molecules. In the process of dehydration and subsequent crushing, carbonate sludge particles acquire nanostructural features, primarily associated with the appearance of porosity. The physicochemical parameters of dehydrated sludge of water treatment at Kazan thermal power plant are presented in Table II.
TABLE II. PHYSICOCHEMICAL INDICATORS OF DEHYDRATED WATER TREATMENT SLUDGE

\begin{tabular}{|c|c|c|}
\hline \multirow{2}{*}{$\begin{array}{c}\text { Physicochemical indicators } \\
\text { of dehydrated water } \\
\text { treatment sludge }\end{array}$} & $<0.09$ & Sludge particle size, mm \\
\cline { 2 - 3 } & 0.876 & 0.85 \\
\hline Bulk density g cm ${ }^{-3}$ & 4.6 & 2.8 \\
\hline Specific surface area, $\mathrm{m}^{2} \mathrm{~g}^{-1}$ & 2.3 & 1.41 \\
\hline Adsorption, $\mathrm{mg} \mathrm{g}^{-1}$ & 82.3 & 89.4 \\
\hline Ash content, $\%$ & 0.71 & 0.66 \\
\hline Humidity, $\%$ & 0.709 & 0.734 \\
\hline Solubility in process water, $\%$ & 0.395 & 0.394 \\
\hline Solubility in process water, $\%$ & & \\
\hline
\end{tabular}

As an additive to fuel, it is advisable to use a finely dispersed fraction of dehydrated carbonate sludge with a particle size of not more than $0.09 \mathrm{~mm}$ with a total content of calcium and magnesium carbonates of at least $85 \%$.

\section{METHODS}

The conditional viscosity (in Engler's degrees, ${ }^{\circ} \mathrm{E}$ ) was determined with the aid of the Engler viscometer VU-M-PHP. To find the kinematic viscosity, the authors used tables of the translation of Engler's degrees into centistokes.

The dynamic viscosity of various types of fuel oil was investigated, using a rotational viscometer Rheomat RM 100. The angular velocity of rotation of the cylinder ranged from 50 to $300 \mathrm{~s}^{-1}$. A torque proportional to the tangential stress in the annular gap was determined and was converted into an electrical signal. The viscosity values were calculated using an integrated microprocessor that analyzes the changes in the torque and shear rate. The work of the viscometer was controlled from a personal computer via the software "VISCO-RM SOFT".

\section{EXPERIMENT}

The experiment was carried out with the help of the Engler viscometer VU-M-PHP, the results of experimental studies of fuel oil M100 brand, with the addition of diproxamine, are presented in Figures 1, 2. The authors investigated the dependence of the solidification temperature of fuel oil on the concentration of diproxamine (Figure 3), and obtained concentration dependences of the conditional viscosity at temperatures $70{ }^{\circ} \mathrm{C}, 80{ }^{\circ} \mathrm{C}$ and $90{ }^{\circ} \mathrm{C}$ (Figure 4), giving an idea of the magnitude of the reduction in the conventional viscosity of fuel oil in the presence of an additive.

In the next series of experiments, we studied the effect of carbon nanotubes of the carbon nanomaterial "Taunit" on the rheological characteristics of furnace fuel oil. The concentration dependences of the conditional viscosity of fuel oil with the addition of carbon nanotubes of the carbonaceous nanomaterial "Taunit" are shown in Fig. 5. 


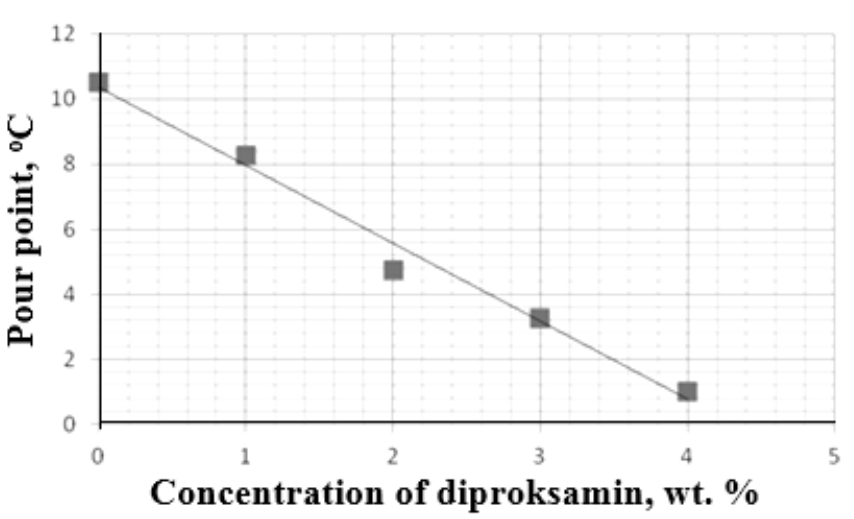

Fig. 3. Dependence of the pour point of M100 brand fuel oil on the concentration of diproxamine

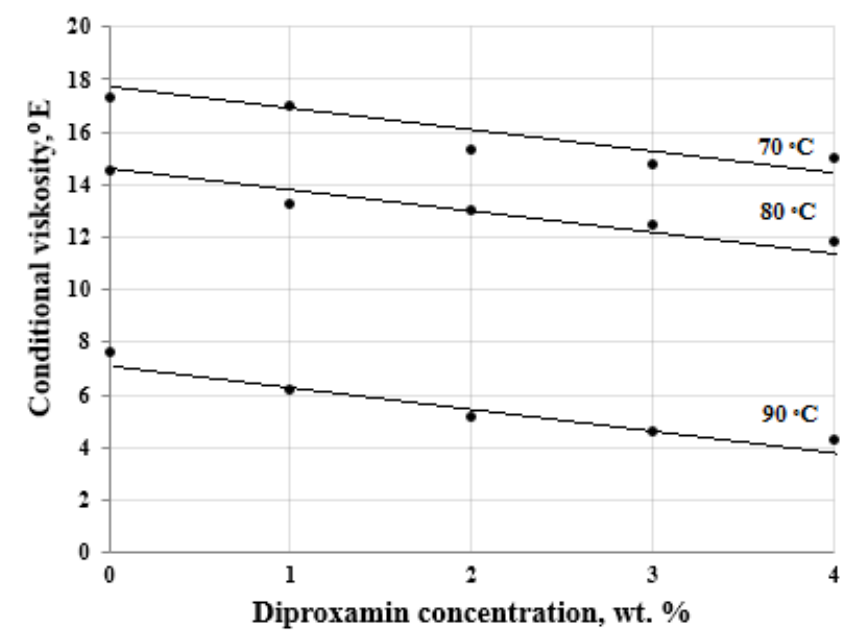

Fig. 4. Dependence of the conditional viscosity of M100 brand fuel oil on the concentration of diproxamine at temperatures of $70{ }^{\circ} \mathrm{C}, 80{ }^{\circ} \mathrm{C}, 90{ }^{\circ} \mathrm{C}$

Investigations of the dynamic viscosity of a composite fuel, which is a mixture of M100 brand fuel oil with diproxamine and a suspension of carbon nanotubes dispersed in diproxamine at temperatures of $65^{\circ} \mathrm{C}$ and $75{ }^{\circ} \mathrm{C}$ are shown in Fig. 6.

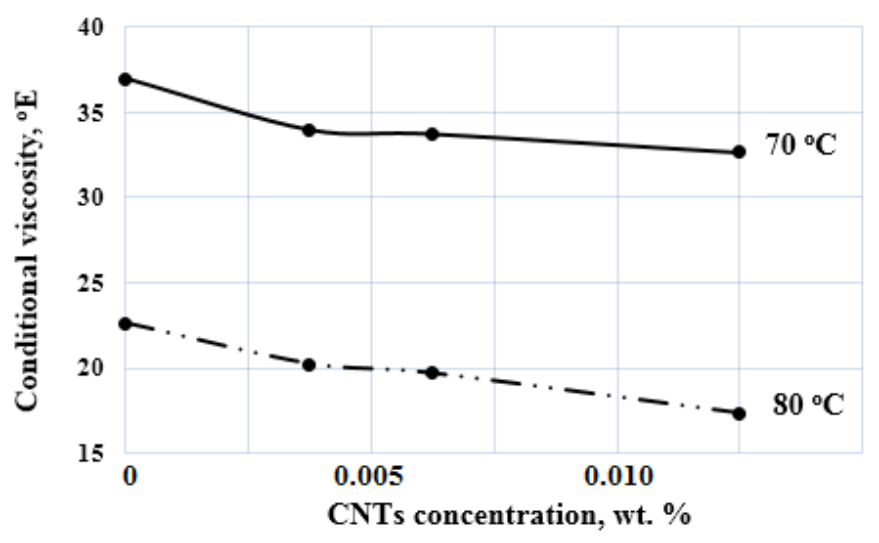

Fig. 5. Dependence of the conditional viscosity of M100 brand fuel oil on the concentration of diproxamine at temperatures of $70{ }^{\circ} \mathrm{C}$ and $80{ }^{\circ} \mathrm{C}$

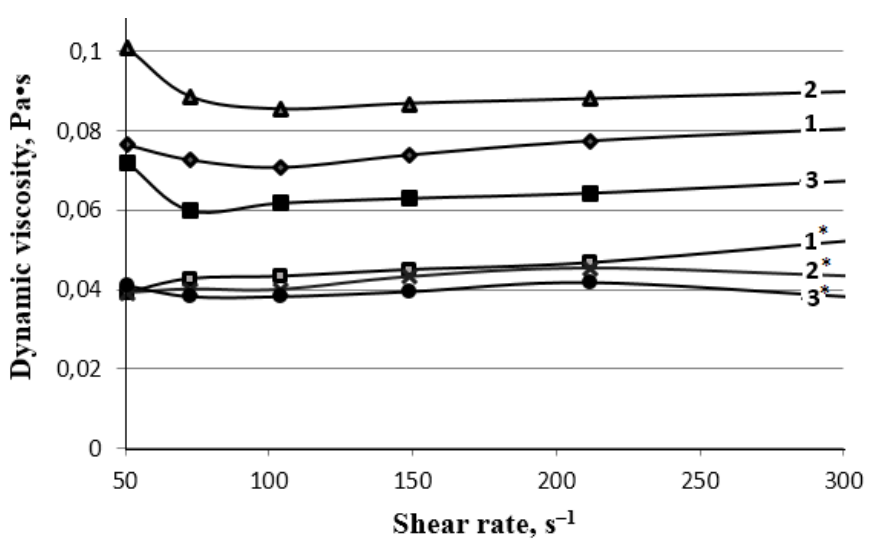

Fig. 6. The dynamic viscosity of M100 brand fuel oil:1, $1^{*}$ - pure fuel oil, 2, $2 *$ - fuel oil +0.5 wt. $\%$ of diproxamine, $3,3 *-$ fuel oil $+0,0125$ wt. $\%$ CNTs +0.5 wt. $\%$ of diproxamine as a function of shear rate at temperatures of $65^{\circ} \mathrm{C}$, $75^{\circ} \mathrm{C}(*)$

Since the addition of carbonate sludge, as it turned out, greatly increases the ash content of fuel oil, studies of concentration dependencies of the conventional viscosity of fuel oil samples with carbonate slurry have not been carried out. The authors preferred an additive concentration of $0.1 \mathrm{wt}$. $\%$. At this concentration, the additive makes it possible to reduce the viscosity of fuel oil (the greatest decrease in dynamic viscosity obtained by us for $70^{\circ} \mathrm{C}$ is shown in Fig. 7) and its pour point, as well as to cut the sulfur content in the emissions and improve the structure of the deposits, increasing the ash content of the fuel oil slightly. The results of industrial tests indicate a decrease in the mass fraction of emitted sulfur oxides by $36.5 \%$ [26].

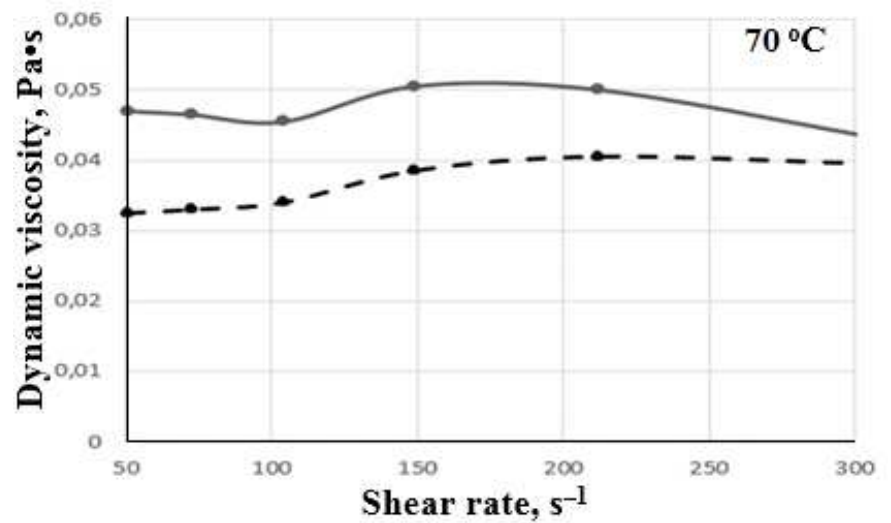

Fig. 7. Dependence of the dynamic viscosity of samples of pure fuel oil M100 brand (solid line) and fuel oil with the addition of $0.1 \mathrm{wt}$. \% carbonate sludge (dashed line) as a function of the shear rate at a temperature of $70{ }^{\circ} \mathrm{C}$

\section{EXPERIMENTAL RESULTS}

Analysis of the concentration dependencies of the pour point and the conditional viscosity of fuel oil samples with diproxamine (Figures 3, 4) showed the possibility of using diproxamine to improve the rheological properties of the fuel. Nevertheless, it should be noted that the effect of diproxamine is more pronounced at high temperatures. This is especially true for economically more advantageous concentrations of 0.5 wt. \% diproxamine. Apparently, this fact is associated with 
an increase in the dynamic viscosity of another sample of fuel oil in the presence of diproxamine at $65{ }^{\circ} \mathrm{C}$ (Figure 6), although the situation changes with an increase in temperature by another $10{ }^{\circ} \mathrm{C}$. Thus, the effect of a positive action of diproxamine in some cases may not be clearly manifested. The addition of carbon nanotubes also led to improved rheological properties of the fuel (Figure 5). Reduction of the conventional viscosity of the heavy fuel oil M100 brand was observed in the entire range of the considered concentrations of carbon nanotubes at $70{ }^{\circ} \mathrm{C}$ and $80{ }^{\circ} \mathrm{C}$. However the expected minimum value, usually observed at a certain concentration of nanoparticles [19], was not achieved by the authors. The authors connect this fact to the very poor quality of the sample, which is clearly manifested by such large values of the conditional viscosity. When finding the optimal concentration of nanoparticles, it is important to get inside a certain concentration range, which depends both on the type of nanoparticles and on the type of dispersion medium and for different types of carbon nanotubes varies from $0.001 \mathrm{wt} . \%$ to 0.5 wt. \% [19].

From Fig. 6, it is seen that even at a temperature of $65^{\circ} \mathrm{C}$, at which the addition of diproxamine alone (0.5 wt. \%) did not lead to a positive effect, the addition of carbon nanotubes dispersed in diproxamine $(0.0125$ wt. $\%$ of CNTs +0.5 wt. \% diproxamine) led to a decrease in dynamic viscosity over the entire range of shear rates. Thus, the use of carbon nanotubes leads to a greater positive effect at lower temperatures.

The addition of $0.1 \%$ wt. \% carbonate sludge (Figure 8) showed good results in improving the rheological properties of fuel oil, but the reduction in its use of the mass fraction of sulfur oxides and other harmful released substances are more important [26].

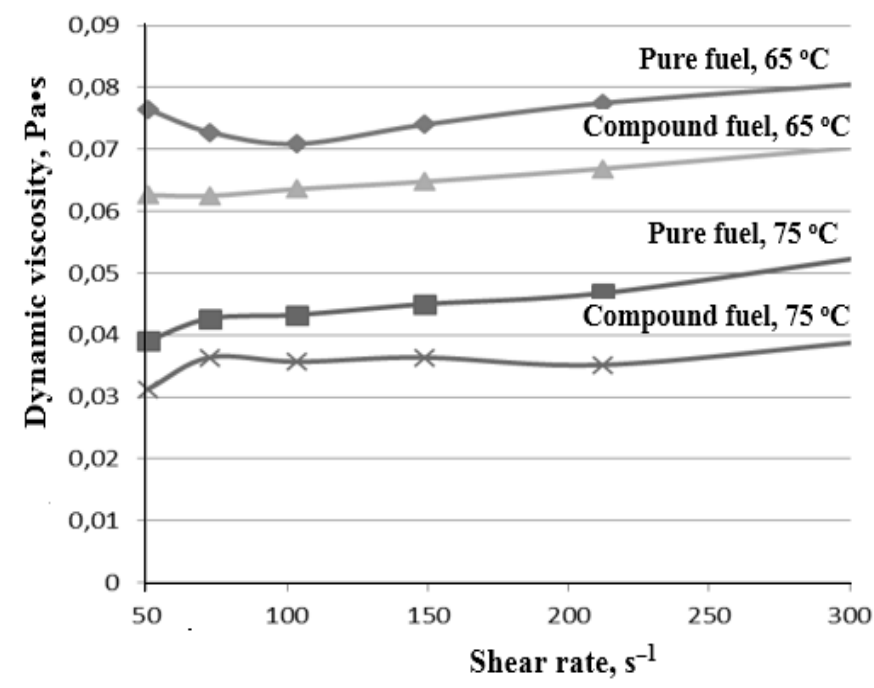

Fig. 8. Dependence of the dynamic viscosity of the M100 brand fuel oil sample (concentration 0.0063 wt. $\%$ CNTs +0.25 wt. $\%$ diproxamine +0.5 wt. $\%$ dehydrated carbonate sludge), as a function of the shear rate at temperatures of $65^{\circ} \mathrm{C}$ and $75^{\circ} \mathrm{C}$

Investigation of the viscosity of the mixed fuel in the presence of both additives (fuel oil M100 + 0.00625 wt. \% CNTs +0.25 wt. \% diproxamine +0.5 wt. $\%$ carbonate sludge) showed the best results, revealing the presence of a synergistic effect from the joint application of these additives
(Fig. 8). The changes, observed in viscosity characteristics, were $10-20 \%$. The authors observed a decrease in viscosity even at low shear rates, which indicates the absence of an explicit structure formation in the petroleum heterogeneous system. In addition, the reduced content in the sample of additional pricing additives - diproxamine and CNTs, should be noted. Despite the fact that the concentration of carbonate sludge is too high (leads to an unjustified increase in the ash content of the fuel), the presence of a synergistic effect can also be expected for its lower concentrations.

The results obtained are well described by the proposed in reference [19] for bitumen and later developed in reference [13] the concept of heterospheres - the additional structure formation of the dispersion medium around nanoparticles in liquid heterogeneous systems. The usual behavior of fuel oil is caused by the emergence of a structural network linking large associates of molecules of high-molecular alkanes or tarasphaltene components. The introduction of nanoparticles (carbon nanotubes) changes the internal structure of the fuel. Nanoparticles play the role of structure-forming centers around which, under the influence of excess surface energy of nanoparticles, ordered layers of matrix material components are formed. At some sufficiently small concentration of nanoparticles, almost complete structuring takes place in the bulk of the sample. In this case, the heterospheres become separated by thin interlayers of the remaining less dense part of the dispersion medium. In this case, the sliding plane from the applied deformation will occur precisely to this interlayer, leading to the appearance of a stratified shear flow and, accordingly, to a sharp decrease in the viscosity for a small concentration region.

A subsequent increase in the concentration of nanoparticles leads to the coupling of heterospheres and an increase in the kinematic viscosity to the initial, and, in some cases even, to greater values. The effect of shear stress in the study of dynamic viscosity in the region of the coupling of heterospheres should lead first to the partial destruction of heterospheres, and, at high shear rates, to their complete destruction. This process should be accompanied by a decrease in the viscosity to values, characterizing the dispersion medium in the absence of nanoparticles.

It should be noted that the concept of the emergence of heterospheres can be extended to any liquid heterogeneous systems containing nanoparticles, despite the fact that the forces, leading to aggregation of molecules, can have different nature depending on the composition and complexity of the systems under consideration, the polarity of the molecules involved in its composition, the presence of certain functional groups, etc. For the existence of an additional structure formation, only two points are important: the very fact of introducing nanoparticles with excess surface energy into a heterogeneous system and the possibility of moving and associating molecules around them due to the liquid aggregate state of the system. Therefore, the behavior of completely different systems turns out to be similar.

It should also be noted that the anisotropic shape of the nanoparticles (primarily for carbon nanotubes, which length after dispergation and ultrasonic action in the authors' samples 
varies on average from 50 to $500 \mathrm{~nm}$ with an average nanotube diameter of $15-20 \mathrm{~nm}$ ) can lead to an incompletely formed spherical shape. However all the above-mentioned arguments remain valid except for the possibility of free rotation of heterospheres. In this case, one should expect only the turning of the formations and their orientation in the direction of the action of the forces, which does not interfere in stratified shear flow.

The proposed concept of heterosphere - regions of the additional structure formation of a dispersive medium around nanoparticles in a liquid - makes it possible to explain the viscosity changes observed by the authors for heavy fuel oil with the addition of nanostructured formations. Moreover according to this concept, the region of viscosity decrease is related to small concentrations of nanoparticles. The best results were actually observed by the authors at concentrations of CNTs of the order of 0.01 wt. \%. Indirectly, this concept is also confirmed by an increase in the density of fuel samples with carbonate sludge additives in the concentration range where the viscosity decreases (Table 3 ). Thus, the use of the concept of heterospheres and the choice of the correct CNTs dispersing medium for a particular type of hydrocarbon fuel allows us to significantly narrow the search for optimal additive concentrations.

TABLE III. DENSITY OF THE SAMPLE M100 BRAND FUEL OIL

\begin{tabular}{|c|c|c|c|c|c|}
\hline \multirow{2}{*}{ Indicator } & \multicolumn{4}{|c|}{$\begin{array}{c}\text { Concentration of dehydrated } \\
\text { carbonate sludge additive in fuel oil }\end{array}$} \\
\cline { 2 - 6 } & 0 & 0.1 & 0.5 & 1.0 & 1.5 \\
\hline Fuel oil density & 990 & 1014 & 1007 & 996 & 993 \\
\hline
\end{tabular}

The decrease in the viscosity of fuel oil with the addition of dehydrated carbonate sludge can be due to the following mechanism: the paraffin present in the fuel oil (47-49\%) easily crystallizes with decreasing temperature, and at certain sizes and concentrations the paraffin crystals form a spatial structure, as a result fuel loses mobility. The additive introduced modifies the surface of the paraffin crystals, preventing their growth and association, as a result of which the viscosity is reduced throughout the region of the investigated shear rates. It is possible that a mechanism of additional structure formation associated with the nanostructural properties of carbonate sludge particles is also manifested here.

It should also be noted that when adding mineral additives to fuel oil, its ash content increases. Ash content is also increased at the addition of diproxamine. It should be noted that the sample containing carbon nanotubes, on the contrary, had a lower ash content than without them.

\section{CONCLUSIONS}

The authors' studies of the viscosity of fuel oil samples have shown the possibility of improving its rheological properties by introducing various nanostructured additives diproxamine $(0.5$ wt. $\%)$, carbon nanotubes of the carbon nanomaterial "Taunit" $(0.0125 \mathrm{wt} . \%)$ or dehydrated carbonate sludge $(0.1 \mathrm{wt} . \%)$. The existence of a synergistic effect at joint application carbon nanotubes with carbonate sludge was established. Possible mechanisms for changing the viscosity properties of fuel were considered.

\section{ACKNOWLEDGMENT}

The reported study was funded by RFBR according to research project № 16-08-00731-a "The improvement of the operational and the environmental characteristics of liquid organic fuel oils by additives including carbon nanotubes."

\section{REFERENCES}

[1] A.R. Mallick, Practical Boiler Operation Engineering and Power Plant, 3rd ed., Delhi: PHI Learning Pvt. Ltd, 2014.

[2] R.C. Eliot, Boiler Fuel Additives for Pollution Reduction and Energy Saving, New Jersey: Noyes Data Corp., 1978.

[3] A. Groysman, Corrosion in Systems for Storage and Transportation of Petroleum Products and Biofuels: Identification, Monitoring and Solutions, New York: Springer Science \& Business Media, 2014.

[4] J.S. Basha, and R.B. Anand, "Role of nanoadditive blended biodiesel emulsion fuel on the working characteristics of a diesel engine", JRSE, vol. 3, 023106, 2011.

[5] T. Shaafi, K. Sairam, A. Gopinath, G. Kumaresan, and R. Velra, "Effect of dispersion of various nanoadditives on the performance and emission characteristics of a CI engine fuelled with diesel, biodiesel and blendsA review,” Renew. Sust. Energ. Rev., vol. 49, pp. 563-573, 2015.

[6] J.S. Basha, and R.B. Anand, "The influence of nano additive blended biodiesel fuels on the working characteristics of a diesel engine," $\mathrm{J}$. Braz. Soc. Mech. Sci. vol. 35, pp. 257-264, 2013.

[7] V. Sajith, C. B. Sobhan, G. P. Peterson "Experimental Investigations on the Effects of Cerium Oxide Nanoparticle Fuel Additives on Biodiesel, Advances in Mechanical Engineering," vol. 2010, 581407, 2010.

[8] A. Selvaganapthy, A. Sundar, B. Kumaragurubaran, and P. Gopal, "An Experimental Investigation to Study the Effects of Various Nano Particles with Diesel on Di Diesel Engine, " ARPN Journal of Science and Technology, vol. 3 (1), pp. 112-115, 2013.

[9] G.R. Kannan, R. Karvembu, and R. Anand, "Effect of metal based additive on performance emission and combustion characteristics of diesel engine fuelled with biodiesel," Applied Energy, vol. 880 (11), pp. 3694-3703, 2011.

[10] W.M. Yang, H. An, S.K. Chou, S. Vedharaji, and R. Vallinagam, "Emulsion fuel with novel nano-organic additives for diesel engine application," Fuel, vol. 104, pp. 726-731, 2013.

[11] E.R. Zvereva, O.S. Zueva and R.V. Khabibullina. "Improvement of Liquid Organic Fuel Oils Operational Characteristics with Additives," Materials Science Forum, vol. 870, pp. 666-670, 2016.

[12] D. Schuster, Encyclopedia of Emulsion Technology: Applications, Boca Raton: CRC Press, 1985.

[13] E.R. Zvereva, O.S. Zueva, R.V. Khabibullina and A.O. Makarova. "Nanomaterial Effect Study in the Viscosity Characteristics of Fuel Oil and Alternative Fuels Used at Fuel and Energy Complex Enterprises," Journal of Engineering and Applied Sciences, vol. 11, pp. 2950-2954, 2016.

[14] V.F. Shumsky, Yu.S. Lipatov, V.G. Kulichikhin, and I.P. Getmanchuk, "Rheological properties of carbon black-filled blends of liquidcrystalline copolyester with thermoplastic polysulfone," Rheologica Acta, vol. 32 (4), pp. 352-360, 1993.

[15] M.E. Mackay, T.T. Dao, A. Tuteja A., D.L. Ho, B. Van Horn, H.C. Kim, and C.J. Hawker. "Nanoscale effects leading to non-einstein-like decrease in viscosity," Nature Materials, vol. 2, pp. 762 - 766, 2003.

[16] A.A. Pykhtin, P.V. Surikov, L.B. Kandyrin, and V.N. Kuleznev. "Influence of ultrafine fillers on the properties of low-molecular liquids and compositions based on epoxy oligomers," Fine Chemical Technologies. vol. 8 (4), pp. 113-117, 2013. 
[17] V.G. Kulichikhin, A.V. Semakov, V.V. Karbushev, N.A. Platé, and S.J. Picken. "The chaos-to-order transition in critical modes of shearing for polymer and nanocomposite melts," Polymer science. Series A., vol. 51 (11), pp. 1303-1312, 2009.

[18] A.Ya. Malkin, and V.G. Kulichikhin, "Structure and rheology of highly concentrated emulsions: a modern look," Russian chemical reviews, vol. 84 (8), pp. 803-825, 2015.

[19] T.V. Mokochunina, "Upgrading modification of oil refining products by carbon nanoparticles," Ph.D. Dissertation in Engineering, Moskow, 2015.

[20] H.S. Jung, A. Miller, K. Park, and D.B. Kittelson. "Carbon nanotubes among diesel exhaust particles: real samples or contaminants?, " J. Air. Waste Manag. Assoc., vol. 63 (10), pp. 1199-1204, 2013.

[21] P. Tewari, E. Doijode, N.R. Banapurmath, and V.S. Yaliwal, "Experimental Investigations on a Diesel Engine Fuelled with Multiwalled Carbon Nanotubes Blended Biodiesel Fuels," IJETAE, vol. 3 (3), pp. 72-76, 2013.
[22] W. Zhang, H. Zhang, J. Xiao, Z. Zhao, M Yua. and Z. Li, "Carbon nanotube catalysts for oxidative desulfurization of a model diesel fuel using molecular oxygen," Green Chem., vol. 16, pp. 211-220, 2014.

[23] W. Zhang, H. Zhang, J. Xiao, Z. Zhao, M Yua. and Z. Li, "Carbon nanotube catalysts for oxidative desulfurization of a model diesel fuel using molecular oxygen," Green Chem., vol. 16, pp. 211-220, 2014.

[24] V.A.M. Selvan R.B. Anand, and M. Udayakumar, "Effect of Cerium Oxide Nanoparticles and Carbon Nanotubes as fuel-borne additives in Diesterol blends on the performance, combustion and emission characteristics of a variable compression ratio engine," Fuel, vol. 130, pp. 160-167, 2014.

[25] E.R. Zvereva, O.S. Zueva, R.V. Khabibullina, G.R. Mingaleeva, G.R. Akhmetvalieva, D.R. Salikhzyanova, and Z.F. Khatmullina. "Effect of Carbon-Nanotube-Based Additives on Rheological Properties of Liquid Boiler Fuel," Chemistry and Technology of Fuels and Oils, vol. 52 (5), pp. 488-494, 2016.

[26] E.R. Zvereva, A.V. Dmitriev, M.F. Shageev, and G.R. Akhmetvalieva, "Results of industrial tests of carbonate additive to fuel oil," Thermal Engineering, vol. 64 (8), pp. 591-596, 2017. 